\title{
Comparison of induction therapies for lupus nephritis
}

Induction therapy for the treatment of proliferative lupus nephritis has traditionally involved cyclophosphamide; however, this agent is associated with various adverse effects, including gonadal toxicity. Researchers have now reported findings from a long-term follow-up comparison of two different induction therapies-azathioprine plus methylprednisolone versus high-dose intravenous cyclophosphamide-and report that treatment with azathioprine and methylprednisolone may be an alternative for particular patients who wish to avoid gonadal toxicity.

The first Dutch Lupus Nephritis Study was initiated in 1995. The researchers randomly allocated 87 patients with proliferative lupus nephritis to receive either azathioprine plus intravenous methylprednisolone and oral prednisone $(n=37)$, or intravenous cyclophosphamide and oral prednisone $(n=50)$. After 2 years, all patients received azathioprine plus oral prednisone. In addition, the researchers regularly obtained clinical and laboratory data and performed renal biopsies in all 87 patients before randomization and in 41 patients after 2 years to evaluate predictors of renal outcome. "Identifying prognostic factors for outcome after immunosuppressive treatment is important for optimizing treatment strategies in individual patients with lupus nephritis", explains Suzanne Arends from the study group.

After a median follow-up of 9.6 years, $11 \%$ of patients reached the primary end point of a sustained doubling in serum creatinine level; the proportion of patients reaching this outcome did not differ between the two treatment groups. Significantly more patients in the azathioprine plus methylprednisolone group than in the cyclophosphamide group reached the secondary end point of renal relapse (38\% versus $10 \%$, $P=0.002)$; however, the researchers found no significant differences in the other secondary end points: end-stage renal disease and death. In addition, no significant differences were found in serum creatinine level or proteinuria at the final visit. "Based on these results, induction treatment with azathioprine and methylprednisolone can serve as an alternative to high-dose intravenous cyclophosphamide in patients with proliferative lupus nephritis who wish to avoid infertility or who have a high risk of premature ovarian failure", states Arends.

\section{$\mathbf{4}$...azathioprine and} methylprednisolone can serve as an alternative to high-dose cyclophosphamide... 77

The researchers also identified clinical and laboratory parameters at baseline and after 2 years that predicted renal outcome but by contrast, found that only baseline renal biopsy parameters were useful in predicting renal outcome. "The present data confirm our previous finding that a protocolized repeat renal biopsy after 2 years does not provide much additional information with regard to long-term renal outcome after immunosuppressive treatment in patients with proliferative lupus nephritis", Arends concludes.

\section{Susan J. Allison}

Original article Arends, S. et al. Long-term follow-up of a randomised controlled trial of azathioprine/ methylprednisolone versus cyclophosphamide in patients with proliferative lupus nephritis. Ann. Rheum. Dis. doi:10.1136/annrheumdis-2011-200384 